\title{
Universiteit
}

Leiden

The Netherlands

\section{Stick-slip motion in spite of a slippery contact: Do we get what we see in atomic friction?}

Krylov, S.; Dijksman, J.A.; Loo, W.A.; Frenken, J.W.M.

\section{Citation}

Krylov, S., Dijksman, J. A., Loo, W. A., \& Frenken, J. W. M. (2006). Stick-slip motion in spite of a slippery contact: Do we get what we see in atomic friction? Physical Review Letters, 97(16), 166103. doi:10.1103/PhysRevLett.97.166103

Version: $\quad$ Not Applicable (or Unknown)

License: $\quad$ Leiden University Non-exclusive license

Downloaded from: https://hdl.handle.net/1887/61320

Note: To cite this publication please use the final published version (if applicable). 


\title{
Stick-Slip Motion in Spite of a Slippery Contact: Do We Get What We See in Atomic Friction?
}

\author{
S. Yu. Krylov, J. A. Dijksman, W. A. van Loo, and J. W. M. Frenken \\ Kamerlingh Onnes Laboratory, Leiden University, P.O. Box 9504, 2300 RA Leiden, The Netherlands
}

(Received 13 July 2006; published 20 October 2006)

\begin{abstract}
Atomic force microscopy provides direct atomic-scale access to friction. In this paper, unexpected and potentially dramatic consequences of the tip elasticity are discussed. Under certain natural conditions an essentially new, nontrivial regime can be entered. Although the tip appears to perform typical stick-slip motion, the tip-surface contact is fully "lubricated" by fast thermal motion of the tip apex. The interpretation of the observations needs to be changed completely in this case.
\end{abstract}

DOI: 10.1103/PhysRevLett.97.166103

Shortly after the invention of the atomic force microscope (AFM) this instrument has been applied [1] as a friction force microscope (FFM). This seemingly trivial extension of the AFM to the lateral direction has enjoyed great interest [2], since it is believed to provide direct atomic-scale access to the phenomenon of friction. Experiments with atomic resolution typically show periodic, sawtoothlike behavior of the lateral force known as stick-slip. The FFM tip is thought to be held periodically in lattice positions of the surface, which is easily modeled using a quasistatic approach, first proposed by Prandtl [3] and often referred to as the Tomlinson model [4-6]. An object (the tip) is considered to move in a periodic potential field formed by the substrate lattice, while being dragged along the surface by an external spring (the cantilever), which is at the same time used to measure the lateral force experienced. Generalizations of the model, from dynamical modeling to nonequilibrium statistical mechanics [7], have advanced our understanding of atomic-scale friction, e.g., its velocity dependence, transitions from stick-slip to other regimes, and the role of thermal effects [4,8-10].

A basic problem, which has not been fully recognized yet, is concerned with flexibility of the tip. At first glance, the tip seems a very hard object. However, the spring constant measured in experiments is not equal to the stiffness $K$ of the cantilever. Usually it is much lower and it can be presented as $K_{\text {eff }}=\left(K^{-1}+k^{-1}\right)^{-1}$, with $K_{\text {eff }}$, and hence $k$, typically of the order of $1 \mathrm{~N} / \mathrm{m}$, i.e., of the order of stiffness of atomic bonds. There is no other way than to associate $k$ with the flexibility of the tip, which hence turns out to be even softer than most cantilevers. A correction for this tip flexibility can be easily introduced in the quasistatic description of stick-slip, but possible changes in dynamics of the system have only attracted attention recently. Flexibility of the tip brings an additional channel of dissipation into the system that can lead to a nonmonotonous velocity dependence of friction [9]. Another, rather subtle manifestation of the tip flexibility, as concluded from simulations of a two-spring system [11], is in the duration of slip events.

In this Letter, we show unexpected and potentially dramatic consequences of the tip elasticity in combination
PACS numbers: 68.35.Af, 07.79.- $-\mathrm{v}, 46.55 .+\mathrm{d}, 81.40 . \mathrm{Pq}$

with thermal effects in the tip-apex-surface contact: under certain realistic conditions they can lead to substantial changes in the observed lateral forces.

We interpret the atomically low values of the effective spring constants as the signature of a small group of atoms at the tip apex. Below we start with an estimate of the relevant characteristic frequency of a typical FFM tip apex, concluding it to be very high, of the order of several GHz or even larger. We further show that the low-frequency response of the cantilever provides a very indirect-averaged-view of the complex dynamics of the tip-apex motion. As a result, what the FFM measures can be very different from what the tip apex does. In particular, rapid thermal motion of the tip apex can lead to a very slippery contact with the surface. A strongly counterintuitive scenario- "stuck in slipperiness" - is predicted to apply under certain natural conditions. The cantilever can exhibit stick-slip motion, thus revealing a nonzero mean friction force, while the tip-surface contact is completely "thermally lubricated" by fast activated jumps of the tip apex, back and forth, between the surface potential wells. Importantly, in this case the apparent surface corrugation (derived from experimental data using the traditional onespring interpretation) can be substantially smaller than the real one. Moreover, the transition from stick-slip motion to continuous, low-dissipative sliding, as predicted by the Tomlinson model and recently observed experimentally $[5,6]$, can take place at a higher value of the relative surface corrugation than expected.

We start with a simple description, in which the tip consists of $N$ rigid atomic layers, and introduce spring constants $k_{i}$ characterizing tangential displacement between layers $i$ and $i+1$. For simplicity, we imagine only shearlike interlayer motion which makes $k_{i}$ proportional to the number of atoms in the layer and to the mass $m_{i}$. The ratio $\frac{k_{i}}{m_{i}}$ then remains the same all along the tip and $\frac{k_{i}}{m_{i}} \simeq \frac{k_{\text {at }}}{m_{\text {at }}}$, with $k_{\text {at }}$ and $m_{\text {at }}$ the characteristic stiffness of an atomic bond and the atomic mass. Recalling the classical problem [12] of normal vibrational modes in a long linear array of objects with nearest neighbor interactions, one finds frequencies $\nu_{j}=\nu_{0}\left|\sin \left(\frac{\pi}{2} \frac{N-j}{N}\right)\right|$, with $j=1,2, \ldots,(N-1)$, and, in our case, with $\nu_{0}=\frac{1}{\pi} \sqrt{\frac{k_{\text {at }}}{m_{\text {at }}}}$. Consequently, the high- 
est eigenfrequency of the tip is of the order of the atomic vibration frequency, $\nu_{\max } \approx \nu_{0} \sim 1 \mathrm{THz}$, and this result is independent of length and shape of the tip. For the lowest frequency, taking a typical tip length $100 \mu \mathrm{m}$, and hence $N \sim 10^{6}$, one finds $\nu_{\min } \sim 1 \mathrm{MHz}$. Specific values of the lower frequencies depend on boundary conditions and on the tip shape. Importantly, the spectrum of relevant intratip vibrations ranges from $\mathrm{MHz}$ to $\mathrm{THz}$, and this entire window is well above the typical frequency of the cantilever $(1-200 \mathrm{kHz})$. Moreover, there are two reasons for higher frequencies to dominate the motion of the tip apex. One is that the density of states is higher at higher frequencies. Second, the apex region is associated with low $k_{i}$ values, and thus large thermal amplitudes, whereas the low $m_{i}$ values keep the frequencies high.

We have also taken a more quantitative approach by computing the lowest normal modes for several relevant tip shapes, using finite element analysis [13]. In agreement with the above estimates, they are in the MHz range; for the example, shown in Fig. 1(a), $\nu_{\min }=6.6 \mathrm{MHz}$. Figure 1(b) shows the elastic deformation of the same tip under a static tangential force applied at its end. As expected, the main deformation is at the end of the tip, which indicates that the effective mass that actually probes the surface is very small so that the associated effective frequency should be high. The result depends on the detailed atomic structure of the tip apex. Suppose the tip apex is a pyramid, with $n^{2}$ atoms in the final terrace, $(n+1)^{2}$ in the second atomic layer, $(n+2)^{2}$ in the third, etc. Returning to the model introduced above, one calculates the effective stiffness of the tip, $k$, as determined by the series of springs $k_{i}, k^{-1}=$ $\sum_{i=1}^{N} k_{i}^{-1}$. The sum converges rapidly and the result is practically independent of the full length of the tip, but it depends on the size $n$ of the first layer. The typical experimental result, $k \sim k_{\text {at }}$, is reproduced with $n \sim 3$, i.e., for about ten atoms in contact with the surface, and the final
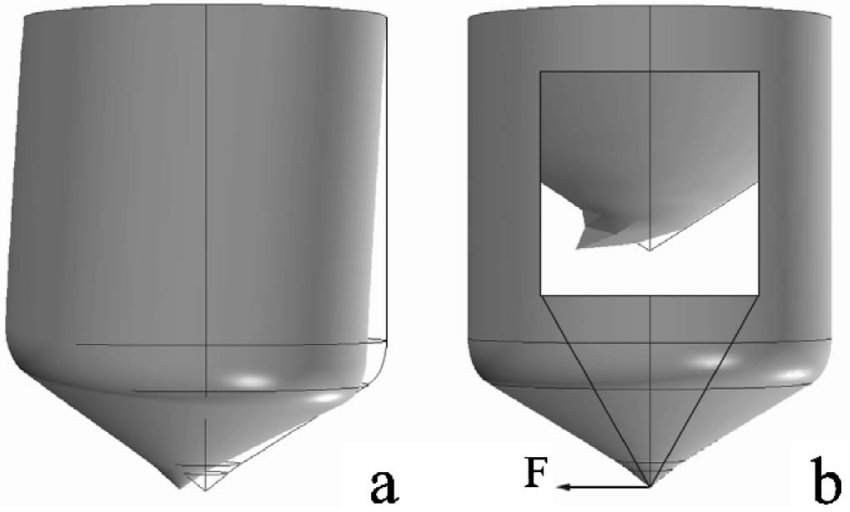

FIG. 1. Elastic deformation of a tip of full length $100 \mu \mathrm{m}$, conical part length $25 \mu \mathrm{m}$, and base diameter $125 \mu \mathrm{m}$, due to vibration in its lowest normal mode, $\nu_{\min }=6.6 \mathrm{MHz}$ (a), and due to tangential force applied at its end (b). For visualization, tangential displacements of all points have been proportionally magnified. Inset shows the apex region magnified. value of $k$ is approached within $2 \%$ at the first 100 atomic layers. The corresponding effective mass is $m_{\mathrm{eff}} \sim 4 \times$ $10^{5} m_{\text {at }}$ (upper estimate), and the associated effective frequency of the tip $\nu_{t}=\frac{1}{2 \pi} \sqrt{\frac{k}{m_{\text {eff }}}}$ is several $\mathrm{GHz}$ (lower estimate). It comes as no surprise that the effective frequency lies in the middle of the range of normal frequencies of the tip and this result looks rather general. Direct observations of such FFM tip frequencies are still lacking, but experiments on nanometer sized metal spheres show a breathing mode in the range of tens of GHz [14], which is in the estimated frequency range.

An important consequence of the tip flexibility is the coexistence of two relevant types of motion: motion of the tip as a whole (together with the cantilever) and displacements of its apex relative to the position of the center of mass. Since the coupling to the surface is determined by the position of the apex, one has to solve not just one equation of motion (as usual) but at least two coupled equations, one for the tip and the other for its deformation. Fortunately, the problem is simplified by a pronounced hierarchy between the characteristic frequencies of the cantilever $\left(\nu_{c}\right)$ and the tip apex: $\nu_{t} \gg \nu_{c}$ as shown above. It is hence useful to consider motion of the tip apex for a given position of the center of mass (c.m.) of the tip. Then one can consider the dynamics of the tip as a whole, with an effective tip-surface interaction averaged over the fast motion of the apex. At this second stage the problem is reduced to the usual 1-spring-1-mass form. Actually, the situation is complicated by thermal effects which can manifest themselves in a nontrivial way.

Assuming a one-dimensional geometry, the total potential energy of the system can be written as

$$
U(X, x, t)=\frac{K}{2}(V t-X)^{2}+\frac{k}{2}(X-x)^{2}+U_{s}(x),
$$

with $X$ and $x$ the coordinates of the c.m. of the tip and its apex, respectively, and with $V t$ the position of the support moving with the scanning velocity $V$. The tip-surface interaction is assumed to be sinusoidal, with amplitude $U_{0}$ and period $a, U_{s}=\frac{U_{o}}{2}\left[1-\cos \left(\frac{2 \pi x}{a}\right)\right]$.

For fixed positions of both the support and the c.m., $U(x)$ given by (1) determines the motion of the apex. Its possible behavior depends on two important parameters. One is the relative surface corrugation experienced by the apex, $\gamma=$ $\frac{2 \pi^{2} U_{0}}{k a^{2}}$. It is reminiscent of the characteristic parameter of the one-spring theory, $\gamma_{\text {eff }}=\frac{2 \pi^{2} U_{0}}{K_{\text {eff }} a^{2}}$ (see, e.g., [6] ). If $\gamma<$ 1 , the potential function $U(x)$ has only one minimum, for any position $X$ of the c.m., but it has two or more wells when $\gamma>1$ (see Fig. 2). This brings an essentially new time scale into the problem, the time scale of thermally activated "jumps" of the apex between wells (at a fixed position of the c.m.). If $\gamma$ is not too close to unity, the characteristic rate of such jumps $\left(r_{a}\right)$ can be estimated as $r_{a} \sim \nu_{t} \exp \left(-\frac{U_{0}}{k_{B} T}\right)$. 


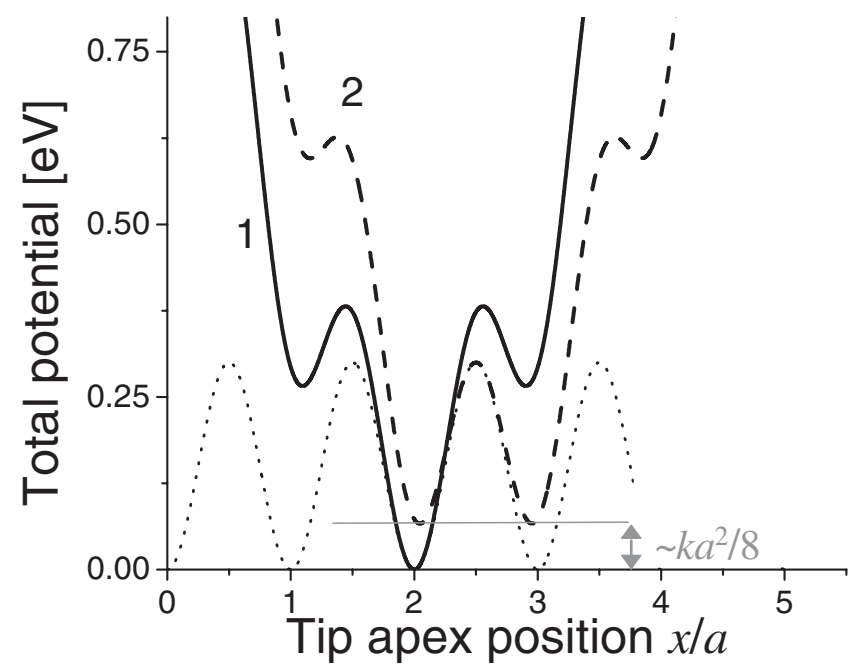

FIG. 2. Total potential $U$ vs tip-apex position $x$ for $U_{0}=$ $0.3 \mathrm{eV}, \gamma=10$ and for two positions of the tip's center of mass, $X=2 a$ (1) and $X=2.5 a(2)$.

In cases $\gamma<1$ (single well) and $\gamma>1, r_{a} \ll \nu_{c}$ (multiple well, negligible rate of thermally activated hops) the situation is simple. The apex resides in a well until the displacement of the c.m. forces it to move to the neighboring well. Replacing $x$ by its mean value and requiring $\frac{\partial U}{\partial X}=$ 0 , we can reduce the total potential (1) to the traditional one-spring form, $U=\frac{K_{\text {eff }}}{2}(V t-\bar{x})^{2}+U_{s}(\bar{x})$, with $K_{\text {eff }}=$ $\left(K^{-1}+k^{-1}\right)^{-1}$. This response is equivalent to that of a tip with coordinate $\bar{x}$, connected to the support by a single (effective) spring $K_{\text {eff }}$, so that the earlier developed theoretical approaches can be applied.

If $r_{a} \sim \nu_{c}$ the situation changes dramatically. Thermal motion of the apex in contact with the surface cannot be averaged out, and one has to solve two coupled equations of motion, as mentioned above, taking explicitly into account activated jumps of the apex. One anticipates the tipcantilever system to exhibit stochastic behavior, an irregular stick-slip. Taking $\nu_{t} \sim 10 \mathrm{GHz}$, following the above estimate, and considering a cantilever with, say, $\nu_{c} \sim$ $10 \mathrm{kHz}$, one observes the situation $r_{a} \sim \nu_{c}$ to take place at room temperature for surface corrugations $U_{0} \sim 0.4 \mathrm{eV}$. Note that the window for this stochastic regime is rather narrow. In view of a strong dependence of $r_{a}$ on $\frac{U_{0}}{T}$, even a small change of $U_{0}$ or $T$ moves the system either to the previous or to the next regime.

When $r_{a} \gg \nu_{c}$ we are in a curious situation which forms the main message of this Letter. The apex jumps frequently back and forth between the accessible surface potential wells, thus making the tip-surface contact very slippery. At first glance, the surface corrugation as experienced by the c.m. is expected to be averaged out, resulting in continuous sliding along the surface. However, in the following we show that the tip can still exhibit stick-slip motion, being, as it appears, stuck in the slipperiness of the tip-substrate interaction.
To demonstrate this counterintuitive behavior we first observe that, since $r_{a} \gg \nu_{c}$, there is always an equilibrium distribution of the apex over all potential wells involved (see Fig. 2). As a result, the total potential (1), after averaging over fast motion of the apex, takes a one-spring form, $\bar{U}(V t, X)=\frac{K}{2}(V t-X)^{2}+\bar{U}_{s}(X)$, with the effective tip-surface interaction $\bar{U}_{s}(X)=c^{-1} \int U^{*} \exp \left(-\frac{U^{*}}{k_{B} T}\right) d x$, with $U^{*}=\frac{k}{2}(X-x)^{2}+U_{s}(x)$ and $c=\int \exp \left(-\frac{U^{*}}{k_{B} T}\right) d x$. Examples are plotted in Fig. 3. Indeed, $\bar{U}_{s}(X)$ turns out to be periodic, with period $a$ of the surface lattice, but it has a specific, nonsinusoidal shape, and its corrugation is substantially reduced with respect to the true surface corrugation $U_{0}$. The underlying physics is easy to understand. The apex spends some time in every one of the potential wells shown in Fig. 2. The effective interaction reflects a weighted average over all these apex positions. When the c.m. position coincides with one of the wells in the surface potential [see Fig. 2(1)], that will strongly dominate the average and $\bar{U}_{s}(X) \simeq U_{s}(X)$. When $X$ is midway between two surface wells [Fig. 2(2)], both wells will contribute equally and $\bar{U}_{s}(X) \simeq U_{s}\left(X-\frac{a}{2}\right)+\frac{k}{2}\left(\frac{a}{2}\right)^{2}$. Thus, the effective potential reaches maxima and minima where the true surface potential does and the maximum variation (corrugation) in $\bar{U}_{s}$ is approximately $\frac{1}{8} k a^{2}$. More accurately, this result is correct if $\gamma$ is large enough and temperature is not too high. In this way one understands why $\bar{U}_{s}$, as seen from Fig. 3, varies significantly with $k$ and with $a$, but it is only weakly dependent on $U_{0}$ and $T$. The situation is curious indeed: the effective tip-surface interaction is created by rapid thermal motion of its apex, which uses the substrate

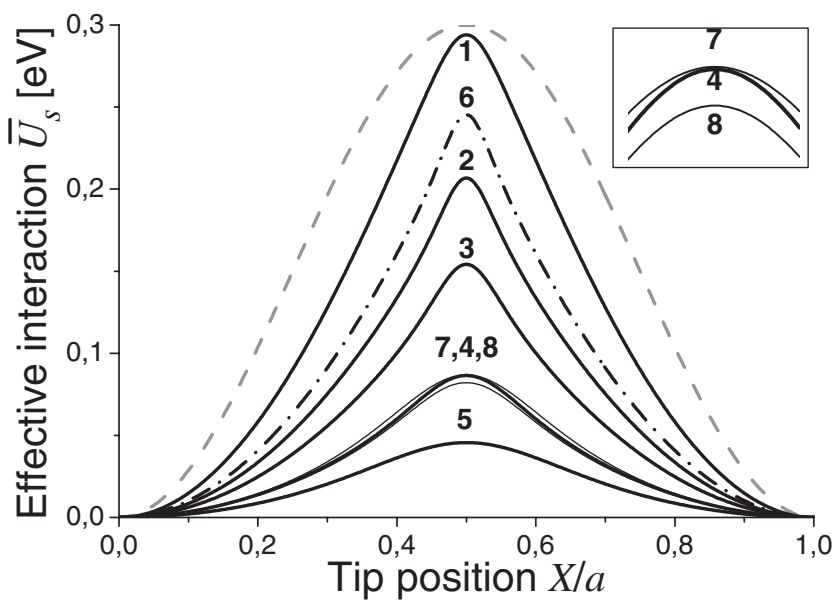

FIG. 3. Effective tip-surface interaction $\bar{U}_{s}$ as a function of the tip position $X$ for $U_{0}=0.3 \mathrm{eV}, a=0.25 \mathrm{~nm}$, and $T=300 \mathrm{~K}$. Curves $1,2,3,4$, and 5 correspond to $k=15.2,6,4,2$, and $1 \mathrm{~N} / \mathrm{m}$ ( $\gamma=1,2.5,3.8,7.6$, and 15.2 , respectively). 6 - the same as 4, but with $a=0.5 \mathrm{~nm} ; 7$ - the same as 4 , but with $T=$ $400 \mathrm{~K} ; 8$ - the same as 4 , but with $U_{0}=0.2 \mathrm{eV}$. The dashed upper line represents the true surface potential. Only one period is shown. 


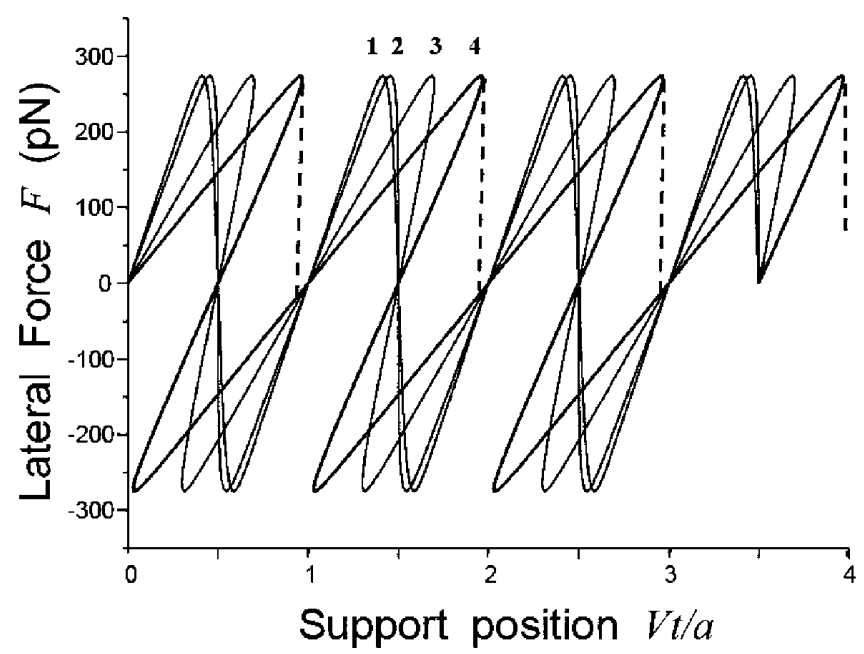

FIG. 4. Lateral force $F$ as a function of the support position $V t$, for $U_{0}=0.3 \mathrm{eV}, a=0.25 \mathrm{~nm}$, and $k=3.5 \mathrm{~N} / \mathrm{m}$. Force loops $1,2,3$, and 4 correspond to $K=\infty, 26,4$, and $2 \mathrm{~N} / \mathrm{m}$, respectively. Dashed lines indicate mechanical slips of the tip (shown for case 4 only).

lattice as a template but produces a result nearly independent of the true surface corrugation.

Further, one can follow the traditional scheme, but with the spring $K$ completely determined by the cantilever, and with the effective tip-surface interaction $\bar{U}_{s}(X)$. Here we concentrate on the most principal observables of the FFM, leaving out possible further thermal and dynamical effects of the types earlier discussed [4,8-11]. For the lateral force measured in experiments, $F=K(V t-X)$, requiring $\frac{\partial \bar{U}}{\partial X}=$ 0 , one easily derives the equation $\left.\frac{d \bar{U}_{s}}{d X}\right|_{X=V t-F / K}-F=0$. Examples for its numerical solution are shown in Fig. 4. The occurrence of stick-slip is indicated by unstable branches of $F(V t)$, which are seen to appear for sufficiently small values of $K$ and are absent for harder cantilevers. The transition from stick-slip to continuous, low-dissipative sliding is a matter of great interest $[5,6]$. Within the usual one-effective-spring approach, the transition takes place when the dimensionless parameter $\gamma_{\text {eff }}=\frac{2 \pi^{2} U_{0}}{K_{\text {eff }} a^{2}}$ is equal to unity. This classical result is not valid anymore if the system is in the regime $r_{a} \gg \nu_{c}$, considered here. For the example in Fig. 4, the critical value of $K$ is $25 \mathrm{~N} / \mathrm{m}$. With $k=3.5 \mathrm{~N} / \mathrm{m}, U_{0}=0.3 \mathrm{eV}$, and $a=0.25 \mathrm{~nm}$, as used in our calculations, the corresponding value of $\gamma_{\mathrm{eff}}$ is 4.9, which is indeed quite different from unity.

An important FFM observable is the maximum value of the lateral force. Within the traditional analysis it is directly related to the surface corrugation, $F_{\max }=\frac{\pi U_{o}}{a}$. Actually, it can be considerably smaller. For the example shown in Fig. $4, F_{\max } \approx 270 \mathrm{pN}$. From this value one derives an apparent surface corrugation of $U_{0}=\frac{F_{\max } a}{\pi} \approx$
$0.15 \mathrm{eV}$, which is a factor of 2 lower than the value of 0.3 eV actually used in Fig. 4.

In summary, the bending motion of the FFM tip, being mainly associated with a small group of atoms at its apex, is very rapid. For a sufficiently weak surface corrugation and soft cantilever, this brings the system into an essentially new regime, in which the tip-surface contact is absolutely slippery, but the cantilever exhibits, nevertheless, seemingly typical stick-slip motion. Basic FFM observables are changed in this case. Our calculations have been based on a lower estimate of the effective frequency of the tip. The preexponential factor of thermally activated motion of the apex is not completely determined by the vibrational frequency. It can be substantially increased by a collective, entropic effect analogous to that recognized in diffusivity of large surface clusters [15]. In this case the new regime of being "stuck in slipperiness" can extend to higher surface corrugations and harder cantilevers. Several of the basic parameters involved, e.g., the characteristic frequency $\nu_{t}$ of the tip apex, are not known a priori. This makes it difficult to conclude at this stage which experiments have been affected by this phenomenon and which have not. We hope that our results will stimulate further experimental and theoretical work in this field.

This work was supported by the Foundation for Fundamental Research on Matter (The Netherlands).

*Permanent address: Institute of Physical Chemistry, Russian Academy of Sciences, Leninsky prospect 31, 119991 Moscow, Russia.

Email address: krylov@redline.ru

[1] C. M. Mate et al., Phys. Rev. Lett. 59, 1942 (1987).

[2] R. W. Carpick and M. Salmeron, Chem. Rev. 97, 1163 (1997).

[3] L. Prandtl, ZS. f. angew. Math. u. Mech. 8, 85 (1928).

[4] E. Gnecco et al., Phys. Rev. Lett. 84, 1172 (2000).

[5] M. Dienwiebel et al., Phys. Rev. Lett. 92, 126101 (2004).

[6] A. Socoliuc et al., Phys. Rev. Lett. 92, 134301 (2004).

[7] M. H. Müser, M. Urbakh, and M. O. Robbins, Adv. Chem. Phys. 126, 187 (2003).

[8] O. K. Dudko et al., Chem. Phys. Lett. 352, 499 (2002).

[9] P. Reimann and M. Evstigneev, Phys. Rev. Lett. 93, 230802 (2004).

[10] S. Yu. Krylov et al., Phys. Rev. E 71, 065101(R) (2005).

[11] S. Maier et al., Phys. Rev. B 72, 245418 (2005).

[12] See, e.g., D. A. Davies, Waves, Atoms and Solids (Longmans, New York, 1978).

[13] W.D. Pilkey, Formulas for Stress, Strain, and Structural Matrices (Wiley, New York, 1994).

[14] M. A. van Dijk, M. Lippitz, M. Orrit, Phys. Rev. Lett. 95, 267406 (2005).

[15] S. Yu. Krylov, Phys. Rev. Lett. 83, 4602 (1999). 